\title{
Cyclic Correlation of Diffuse Reflected Signal with Glucose Concentration and Scatterer Size
}

\author{
Jitendra Solanki ${ }^{1}$, Pratima Sen ${ }^{2}$, Joseph Thomas Andrews ${ }^{1 *}$, Kamal Kishore Thareja ${ }^{3}$ \\ ${ }^{1}$ Applied Photonics Laboratory, Department of Applied Physics, Shri G S Institute of Technology \& Science, Indore, India \\ ${ }^{2}$ Laser Bhawan, School of Physics, Devi Ahilya University, Indore, India \\ ${ }^{3}$ KK Pathology Laboratory, Chanakya Complex, Malwa Mill Circle, Indore, India \\ Email: ${ }^{*}$ jtandrews@sgsits.ac.in
}

Received June 27, 2011; revised August 19, 2011; accepted September 10, 2011

\begin{abstract}
The utility of optical coherence tomography signal amplitude measurement to monitor glucose concentration in tissue phantom and blood samples from human subjects has been explored. The diffusion equation based calculations as well as in-vivo OCT signal measurements confirm a cyclic correlation of signal intensity with glucose concentration and scatterer size.
\end{abstract}

Keywords: Light Scattering; Tomography; Biomedical Optical Imaging; Diabetes; Diffusion

\section{Introduction}

Everyday, almost 150 million people world wide face the problem of diabetic metabolic control. Both the hypo- and hyper-glycemic conditions of patients have fatal consequences and warrant blood glucose monitoring at regular interval. Existing blood glucose monitors can be widely classified into three classes viz., invasive, minimally invasive, and noninvasive. Invasive monitoring require small volume of blood and they are inappropriate for continuous monitoring of blood glucose. Minimally invasive monitors require $1-2 \mathrm{ml}$ of blood for analysis at the same time skin damage of the order of few $\mathrm{mm}$ is also required. On the other hand, noninvasive devices are painless with no skin injury to tissues.

Many research groups are working to develop a real time noninvasive tool for monitoring blood glucose at clinical level. Recent review by Bazaev and Selishchev [1], discuss various noninvasive methods for blood glucose measurement and monitoring. Existing non-invasive blood glucose monitor work on various modalities such as absorption spectroscopy [2], optical activity and polarimetry [3,4], optical coherence tomography (OCT) $[5,6]$, bioimpedance spectroscopy [2], fluorescence [7] etc. The other optical techniques include Raman spectroscopy, absorption [8], and reflectance spectroscopy [9]. However, due to optical interference, poor signal strength, and calibration issues, optical methods still face many challenges [10]. The measurement of blood glucose level using OCT technique exhibits large fluctuations due to motion artifacts or other

${ }^{*}$ Corresponding author. physiological and environmental conditions $[5,6]$.

Optical coherence tomography is a nondestructive technique that examines the internal structure of superficial layers of biological tissues. The backscattered OCT signal from turbid media like blood, tissue, etc. is a diffuse reflected signal which carries information about the scatterer and its environment. It is based on interferometric recording of near-infrared light backscattered from the point of study. Conventionally, in OCT backscattered light is collected, measured, and integrated to assemble images [11]. The objectives of the the present paper are two fold: Firstly, to correlate the glucose concentration with experimentally observed diffuse reflectance signal from OCT. Secondly, to examine the usability of OCT signal intensity for measurement of glucose concentration.

The preceeding section deals with the theoretical analysis where Mie scattering theory and diffusion equation are used to understand the dependence of solute concentration on OCT signal intensity in a turbid medium. In the third section, experimental results are exhibited for various samples of aqueous solutions of glucose dissolved in tissue phantom as the scatterers. The experiment was also performed with blood samples from voluntary blood donors. In the fourth section, the numerical calculations carried out for various glucose concentrations in aqueous solution having different particle sizes as scatterers are presented. These results are compared with the experimental observations and found that glucose concentration and size of scatterers exhibit a cyclic correlation with diffuse reflected signal. 


\section{Theoretical Formulations}

Blood is a turbid medium consisting of extracellular fluid (ECF) and various scatterers e.g. red blood cells (RBC), white blood cell (WBC) etc. Multiple scattering from RBC gives rise to diffusion of light from blood. The average diffuse intensity $\left(U_{d}\right)$ is related to the scattering cross section via the relation [6],

$$
U_{d}(r)=U_{d}(0) \exp \left(-k_{d} r\right) .
$$

Here, $k_{d}\left(=\rho \sqrt{3 \sigma_{a} \sigma_{t r}}\right)$ is the propagation constant of the radiation in ECF with $\rho$ as the number density of scat terers in the medium, $\sigma_{t r}$ is the transport cross section and is given by $\sigma_{t r}\left[=\sigma_{s}(1-g)+\sigma_{a}\right]$ with $\sigma_{s}$ being the scattering coefficient, $g$ is the anisotropy parameter and $\sigma_{a}$ is the absorption coefficient.

The scattering cross sections can be calculated from the Mie scattering theory as [12],

$$
\sigma_{s}=\frac{2 \pi a^{2}}{\alpha^{2}}\left|\sum_{n}(2 n+1)(-1)^{n}\left(a_{n}-b_{n}\right)\right|^{2},
$$

where,

$$
a_{n}=\frac{\psi_{n}(\alpha) \psi_{n}^{\prime}(\beta)-m \psi_{n}(\beta) \psi_{n}^{\prime}(\alpha)}{\xi_{n}(\alpha) \psi_{n}^{\prime}(\beta)-m \psi_{n}(\beta) \xi_{n}^{\prime}(\alpha)}
$$

and

$$
b_{n}=\frac{m \psi_{n}(\alpha) \psi_{n}^{\prime}(\beta)-\psi_{n}(\beta) \psi_{n}^{\prime}(\alpha)}{m \xi_{n}(\alpha) \psi_{n}^{\prime}(\beta)-\psi_{n}(\beta) \xi_{n}^{\prime}(\alpha)}
$$

with $\alpha=k_{d} a, a$ is the scatterer size. $\beta\left(=m k_{d} a\right)$ with $m$ being the relative refractive index of the scatterer (RBC in the present case). Also, $\psi_{n}(\alpha)=\alpha j_{n}(\alpha)$ and $\xi_{n}(\alpha)=\alpha h_{n}^{\prime}(\alpha)$, where $j_{n}(\alpha)$ and $h_{n}(\alpha)$ represent the spherical Bessel and Hankel functions, respectively. With the knowledge of these parameters, we can calculate the path length resolved diffuse reflectance as

$$
R\left(L_{s}\right)=\frac{1}{U_{d}(0)} \times\left.\frac{\partial U_{d}(L)}{\partial L}\right|_{L=L_{s}},
$$

where, $L_{s}\left(=L_{s 0}+\sum_{i=0}^{\infty} L_{s i}\right)$ is the round trip path length estimated from the initial pathlength difference between the sample and reference arms $\left(L_{s 0}\right)$ and the total path length within the sample that accumulates during every $i$ th each scattering event $\left(L_{s i}\right)$ [6]. A theoretical estimate of the OCT signal intensity can be made from the diffuse reflectance. In an OCT set up the interference between the light scattered from the sample arm and reference arm is detected. The light intensity at the detector is given by

$$
I_{d}(\tau)=\left\langle\left|\int_{L_{s}}^{\infty} E_{s}^{\prime}\left(t, L_{s}\right) \mathrm{d} L_{s}+E_{r}(t, \tau)\right|^{2}\right\rangle,
$$

where, $\tau[=\Delta L / c]$ is the time delay corresponding to the round-trip optical path length between two beams and
$E_{s}^{\prime}\left(t, L_{s}\right)$ is the path length resolved field amplitude. $E_{r}$ is field amplitude from the reference mirror.

The OCT signal obtainable from a turbid medium is a convolution of path length resolved diffuse reflectance and the low coherence function arising from the coherence property of the laser source. Accordingly, the OCT signal may be rewritten as

$$
I_{d}\left(L_{s}\right)=I_{r}\left[R\left(L_{s}\right)^{1 / 2} \otimes C\left(L_{s}\right)\right],
$$

where, $I_{d}\left(L_{s}\right)$ is the OCT signal detected by a photodiode, $I_{r}$ is the signals from reference arm. $C\left(L_{s}\right)$ is lowcoherence function of the source.

The change in the intensity of OCT signal vary in accordance with the square root of the variations in the diffuse reflectance. A close observation of Equations (1) to (5) clearly shows that the diffuse reflectance $R\left(L_{s}\right)$ is intimately related to the scattering cross section $\sigma_{s}$, via Equation (2). Also Equation (2) suggests that the scattering cross section is a function of 1) scatterer size and 2) relative refractive indices of the scatterer and the fluid in which the scatterer is dispersed. Consequently, for blood samples one can expect a variation in the OCT signal intensity with the change in the refractive index of ECF as well as change in the size of RBC.

The amplitude of the OCT signal $A\left(L_{s}\right)$ is proportional to the diffuse reflectance $\left(R\left(L_{s}\right)\right)$ and is given by

$$
A\left(L_{s}\right)=\left.\frac{\Delta U_{d}(r)}{U_{d}(r)}\right|_{L=L_{s}} .
$$

It is worth noticing that the RBC cells, which act as source of scatterer in blood have various sizes in the subjects under study. Consequently, the utility of OCT setup for examining glucose concentration in blood requires the knowledge of weighted average of the signal $\left(A W\left(L_{s}\right)\right)$ [13]. Under the Gaussian distribution approximation, it is given by

$$
A W\left(L_{s}\right)=\frac{1}{n} \sum_{n} \frac{\Delta U_{d}(r)}{U_{d}(r)} \exp \left[-\left(\frac{S_{n}-S_{0}}{\Delta S}\right)^{2}\right],
$$

with $S_{0}, S_{n}$ and $\Delta S$ as the size of RBC, mean size of $\mathrm{RBC}$ and variance in size, respectively. The reported values of RBC in healthy subjects are $S_{0} \approx 7.5 \mathrm{~mm}$ and $\Delta S \approx 1$ $\mathrm{mm}$ [13].

\section{Experiment}

The optical coherence tomography setup as shown in $\mathbf{F i}-$ gure 1 used in the present report is developed in house, using a fiber optic Michelson interferometer. We use superluminescent diode (SLD, Superlum SLD-371) as the light source, which is a broad band source with a center wavelength of $841 \mathrm{~nm}$ and line width of $52 \mathrm{~nm}$. The selection of wavelength in the near infrared region has the 


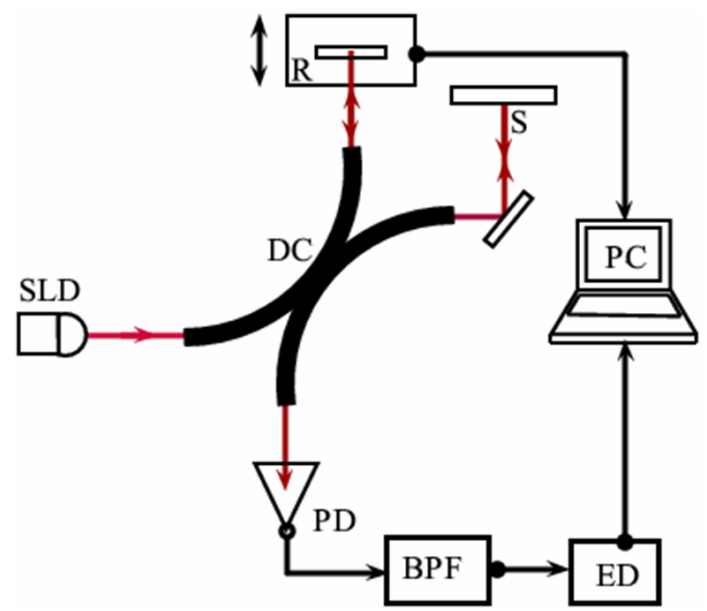

Figure 1. Schematic of the optical coherence tomography setup. SLD-Superluminescent diode; $\mathrm{DC}-3 \mathrm{~dB}$ bi-directional coupler; PD-Photo diode; S-Sample; R-Reference arm with scanning assembly; BPF-Band pass filter; ED-Envelope detector.

advantages of large scattering coefficient and low absorption coefficient in tissues. However, these coefficients are altered when index mismatch occurs between ECF and cells.

\subsection{Sample Preparation}

The primary goal of the present work is to find the correlation between the amplitude of OCT signal with concentration of glucose. Systematic measurements were carried out with the following samples: 1) tissue phantom based as intralipid, and 2) blood samples from voluntary human subjects.

\subsection{Tissue Phantom}

Intralipid is an emulsion of soy bean oil, egg phospholipids and glycerin. Intralipid is widely used in optical experiments to simulate the scattering properties of biological tissues and can be use as a good scatterer as RBC in blood. The major advantages of intralipid are its well known optical properties and the similarity of its microparticles to lipid cell membranes and organelles that constitute the source of scattering in biological tissue [13-17]. We used intralipid as a tissue phantom that provides the backscattered component. Average size of scatterers in intralipid measured using confocal microscope was found to be $3.5 \mathrm{~mm}$.

Using standard approach, we generate glucose concentrations of $0.0 \mathrm{mg} / \mathrm{dl}$ to $200 \mathrm{mg} / \mathrm{dl}$ in $1 \mathrm{ml}$ sample tissue phantom for experimental observations. This range covers hypoglycemic $(<80 \mathrm{mg} / \mathrm{dl})$, normal $(80-120 \mathrm{mg} / \mathrm{dl})$ as well as hyperglycemic $(>120 \mathrm{mg} / \mathrm{dl})$ conditions. A settlement time of 2 minutes was given after addition of glucose to the intralipid solution so that the added glucose could alter the scattering properties of the medium.
Simultaneously, the change in refractive index of the solution was also monitored using Abbe's refractometer. Measurement were carried out using fixed volume $(1 \mathrm{ml})$ of tissue phantom and diluted to $0.01 \%$ in distilled water $(100 \mathrm{ml})$ and concentrated glucose solution prepared by dissolving $200 \mathrm{mg}$ glucose in $100 \mathrm{ml}$ distilled water. The concentration of glucose in intralipid was increased in steps of $10 \mathrm{mg} / \mathrm{dl}$ using U-TEK Chromatography syringe with least count of $5 \mu \mathrm{l}$.

\subsection{Blood Samples}

Blood samples were collected from pathological laboratory. In order to optimize the setup with human subjects, blood samples were collected from various voluntary subjects. Conventional pathological method known as GOD/ POD (Glucose Oxydised/Peroxydised) using semi-automated blood analyzer was used simultaneously to measure the exact value of blood glucose. A smaller part of $(\approx 4$ $\mu \mathrm{l})$ blood taken using a Eppendorf Pipette is placed on the sample arm of the OCT setup.

\section{Results}

To confirm the changes in the optical properties of the solution with increasing glucose concentration in intralipid, Abbe refractometer was used to obtain the refractive index $(=1.407)$ of tissue phantom. As discussed earlier, addition of $50 \mu \mathrm{l}$ glucose to intralipid solution of volume 1 $\mathrm{ml}$ increases the glucose concentration by $10 \mathrm{mg} / \mathrm{dl}$. At the same time, it reduces the index matching between the scatterer and the medium. The measurements of refractive index and scattering coefficients using Abbe refractometer and OCT, respectively were made simultaneously to avoid the temporal fluctuations in values. Results obtained with refractometer are displayed in Figure 2. A linear relation is obtained between the glucose concentration and the refractive index. The data from Figure 2 gives an empirical relation between the glucose concentration and the refractive index.

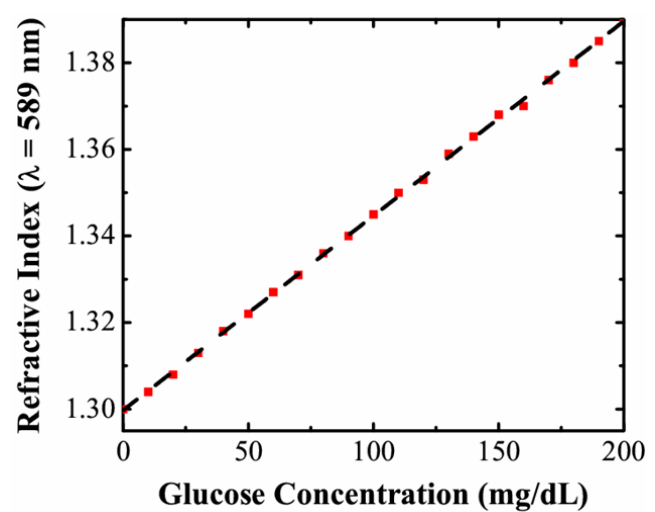

Figure 2. Variation of refractive index of intralipid with Glucose concentration measured using Abbe's refractometer. 
The measured OCT signal amplitudes for various glucose concentrations $(10 \mathrm{mg} / \mathrm{dl}-200 \mathrm{mg} / \mathrm{dl})$ are shown in Figure 3. In order to reduce the noise in OCT signal, we displayed the normalised average area of scattered signal within the coherence length shown as dots in the figure. The OCT signal amplitude is numerically equal to the average diffuse intensity $I_{d}$ defined in Equation (4). The theoretical fit shown as solid line in the Figure 3 is the weighted average as discussed earlier. The results indicate an oscillatory nature of backscattered component of the OCT signal with glucose concentration. These observations with tissue phantom show a cyclic correlation between glucose concentration and OCT signal amplitude and are in good agreement with the theoretically calculated values of diffuse reflectance (solid line). Both the curves exhibit similar nature which confirms the applicability of present theoretical calculations to correlate them with blood glucose levels in human subjects. In order to confirm the same with clinical measurements with human subjects, we collected samples of voluntary donors from pathological laboratory and the results are discussed below. Simultaneous mea-

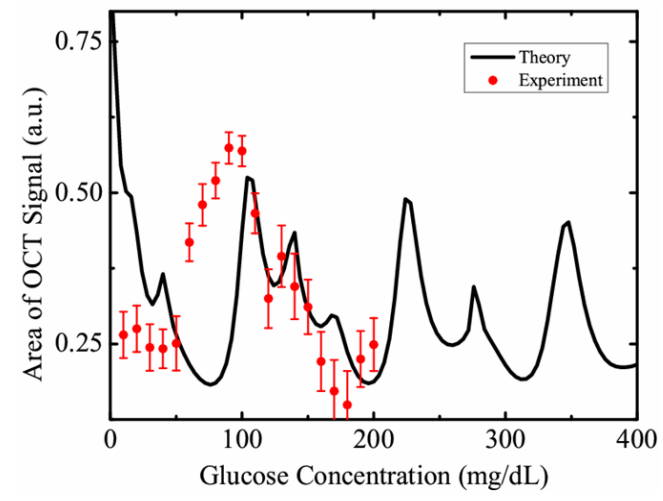

Figure 3. OCT signal amplitude obtained in a sample of tissue phantom with different glucose concentrations are shown as filled circles with error bars. The solid line is the weighted average obtained theoretically using Equation (7).

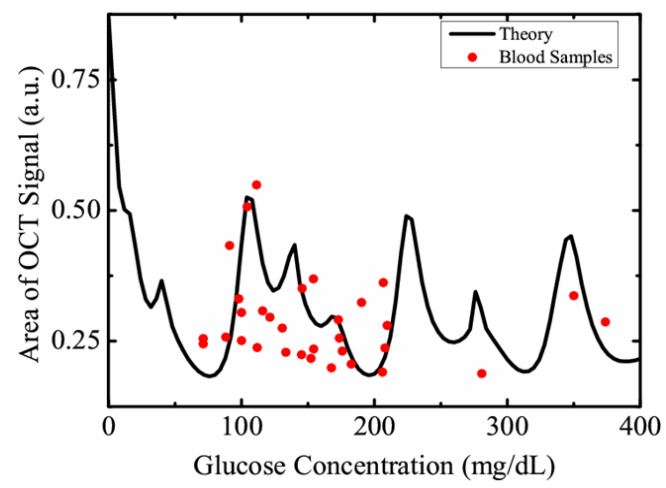

Figure 4. The filled circles represent the amplitude of OCT signal obtained from various blood samples of voluntary donors having different glycemic levels while the solid curve is the weighted average obtained theoretically using Equation (7). surements of blood glucose was carried out using chemical methods as described in previous sections. The correlation between blood glucose level of human subjects and the amplitude of the OCT signal are represented in Figure 4. The curve definitely shows a cyclic correlation, similar to the results predicted from Equation (4) and Figure 3.

We therefore, find that the cyclic correlation of glucose concentration with OCT signal restricts the OCT signal intensity based glucose measurements in human subjects.

\section{Conclusion}

It is well known that the optical properties of tissue vary with glucose concentration and the index mismatch between the ECF and scatterers. There are many factors which will affect the OCT signal intensity. For example the refracttive index of ECF will change with change in any types of soluble impurities (example: urea) and the change in the scatterer size. Accordingly, well defined predictions for glucose concentration measurements using OCT signal intensity are not possible because of the non-uniform size distribution of various particles as well as presence of creatinine, lactic acid, serum albumin, and $\mathrm{NaCl}$, Urea, etc. in blood. Therefore, the results presented here may not be used as a tool for estimation of blood glucose levels, however, it throws light on the understanding of light scattering from blood samples, in the presence of various types of scatterers differing in physical and optical properties.

\section{Acknowledgements}

The authors thank financial support received from UGC and New Delhi and MPCOST, Bhopal, India. The authors also thank Prof. P. K. Sen for fruitful discussions.

\section{REFERENCES}

[1] N. A. Bazaev and S. V. Selishchev, "Noninvasive Methods for Blood Glucose Measurement," Biomedical Engineering, Vol. 41, No. 1, 2007, pp. 42-50. doi:10.1007/s10527-007-0010-9

[2] C. F. Amaral, M. Brischwein and B. Wolf, "Multiparameter Techniques for Noninvasive Measurement of Blood Glucose," Sensors and Actuators B, Vol. 140, No. 1, 2009, pp. 12-16. doi:10.1016/j.snb.2009.04.023

[3] B. Rabinovitch, W. F. March and R. L. Adams, "Noninvasive Glucose Monitoring of the Aqueous Humor of the Eye. Part 1. Measurement of Very Small Optical Rotations," Diabetes Care, Vol. 5, No. 3, 1982, pp. 254-258. doi:10.2337/diacare.5.3.254

[4] G. L. Cote, M. D. Fox and R. B. Northrop, "Noninvasive Optical Polarimetric Glucose Sensing Using a True Phase Measurement Technique," IEEE Transactions on Biomedical Engineering, Vol. 39, No. 7, 1992, pp. 752-756. doi:10.1140/epjc/s10052-010-1303-9 
[5] K. V. Larin, M. Motamedi, M. S. Eledrisi and R. O. Esenaliev, "Noninvasive Blood Glucose Monitoring with Optical Coherence Tomography," Diabetes Care, Vol. 25, No. 12, 2002, pp. 2263-2267. doi:10.2337/diacare.25.12.2263

[6] R. Poddar, S. R. Sharma, J. T. Andrews and P. Sen, "Study of Correlation between Glucose Concentration and Reduced Scattering Coefficients in Turbid Media Using Optical Coherence Tomography," Current Science, Vol. 95, No. 2, 2008, pp. 340-348.

[7] J. C. Pickup, F. Hussain, N. D. Evans, O. J. Rolinski and D. J. S. Birch, "Fluorescence-Based Glucose Sensors," Biosensors and Bioelectronics, Vol. 20, No. 12, 2005, pp. 2555-2565. doi:10.1016/j.bios.2004.10.002

[8] J. R. McNichols and L. G. Cote, "Optical Glucose Sensing in Biological Fluids: An Overview," Journal of Biomedical Optics, Vol. 5, No. 1, 2000, pp. 5-16. doi: $10.1117 / 1.429962$

[9] S. F. Malin, T. L. Ruchiti, T. B. Blank, S. U. Thennadil and S. L. Monfre, "Noninvasive Prediction of Glucose by Near-Infrared Diffuse Reflectance Spectroscopy," Clinical Chemistry, Vol. 45, No. 9, 1999, pp. 1651-1658.

[10] V. Ashok, A. Nirmalkumar and N. Jeyashanthi, "A Novel Method for Blood Glucose Measurement by Noninvasive Technique Using Laser," International Journal of Biological and Life Sciences, Vol. 7, No. 3, 2010, pp. 127132.

[11] V. V. Sapozhnikova, D. Prough, R. V. Kuranov, I. Cicenaite and R. O. Esenaliev, "Influence of Osmolytes on in Vivo Glucose Monitoring Using Optical Coherence Tomography," Experimental Biology and Medicine, Vol.
231, No. 8, 2006, pp. 1323-1332.

[12] A. Ishimaru, "Wave Propagation and Scattering in Random Media," Scattering and Absorption of a Wave by Single Particle, Chapter 2, Vol. 1, Academic Press, New York, 1978, pp. 9-40.

[13] D. N. Mederis and G. R. Minot, "Studies on Red Blood Cell Diameter," Vol. 7, No. 4, 1929, pp. 631-636. doi:10.1172/JCI100247

[14] S. N. Thennadill, J. L. Rennert, B. J. Wenzel, K. H. Hazen, T. L. Ruchti and M. B. Block, "Comparison of Glucose Concentration in Interstitial Fluid, and Capillary and Venous Blood during Rapid Changes in Blood glucose Levels," Diabetes Technology \& Therapeutics, Vol. 3, No. 3, 2001, pp. 357-365. doi:10.1089/15209150152607132

[15] R. Srinivasan and M. Singh, "Laser Backscattering and Transillumination Imaging of Human Tissues and Their Equivalent Phantoms," IEEE Transactions on Biomedical Engineering, Vol. 50, No. 6, 2003, pp. 724-730. doi:10.1109/TBME.2003.812188

[16] H. J. V. Staveren, C. J. M. Moes, J. V. Marle, S. A. Prahl and M. J. C. V. Gemert, "Light Scattering in Intralipid-10\% in the Wavelength Range of $400-1100 \mathrm{~nm}$," Applied Optics, Vol. 30, No. 31, 1991, pp. 4507-4514. doi:10.1364/AO.30.004507

[17] B. F. Kennedy, S. Loitsch, R. A. McLaughlin, L. Scolaro, P. Rigby and D. D. Sampson, "Fibrin Phantom for Use in Optical Coherence Tomography," Journal of Biomedical Optics, Vol. 15, No. 3, 2010, p. 030507-1-3. doi: $10.1117 / 1.3427249$. 